Check for updates

Cite this: Phys. Chem. Chem. Phys., 2017, 19, 30734

Received 10th March 2017.

Accepted 29th October 2017

DOI: $10.1039 / c 7 c p 01553 d$

rsc.li/pccp

\title{
Metal-enhanced Förster resonance energy transfer (ME-FRET) in anthracene/tetracene-doped crystal systems $\dagger$
}

\author{
L. Karnam, ${ }^{\text {ab }}$ L. Brambilla, (DD ${ }^{b}$ M. Del Zoppo (D)*b and C. Bertarelli (D) ${ }^{\text {ab }}$
}

\begin{abstract}
Anthracene tetracene-doped crystals (TA) dispersed in a thin polymer film matrix have been reported as efficient down converting systems based on Förster Resonance Energy Transfer (FRET) which occurs efficiently from donor (anthracene) to acceptor (tetracene) molecules. In this work, we demonstrate that the efficiency of this system can be further improved by a metal-enhanced FRET, which is obtained by incorporating metal nanoparticles into the polymer matrix. We report on the preparation and optical responses of polymeric transparent thin films where dodecanethiol-capped silver or gold nanoparticles are dispersed with TA in a polymethylmethacrylate (PMMA) matrix. A maximum of 11-fold and 6.5-fold enhancement was achieved with silver and gold nanoparticles, respectively. Efficiency is discussed in terms of film morphology and plasmon resonance induced effects.
\end{abstract}

\section{Introduction}

Fluorescence properties of organic compounds in the solid state are extremely important due to their numerous applications in different fields such as organic light-emitting diodes (OLEDs), organic photonics or photoelectronic devices. ${ }^{1-5}$ However, the design of luminescent organic materials in the solid state is problematic since in the aggregated state their emission efficiency generally decreases. In this respect, host-guest systems based on organic molecular crystals have recently provided a good platform for the design of functional materials with enhanced optoelectronic properties such as Resonant Energy Transfer (RET) ${ }^{5-7}$ tunable fluorescence, ${ }^{8}$ and amplified spontaneous emission (ASE). ${ }^{4}$ Among all the reported emitters acene-based compounds have been extensively studied due to their high fluorescence quantum yield and thermal stability. ${ }^{9-11}$

In the recent past, molecular host/guest crystals based on oligoacenes have been shown to promote a very efficient hostto-guest Förster Resonance Energy Transfer (FRET) even at a very low concentration of guest species. ${ }^{5,12}$ Using anthracene/tetracenedoped crystals (TA), we have recently realized thin film luminescent solar concentrators ${ }^{13-15}$ demonstrating an increase in the optical efficiency. ${ }^{16}$ Nevertheless, we noticed that when crystals are

\footnotetext{
${ }^{a}$ Center for Nano Science and Technology@Polimi, Istituto Italiano di Tecnologia, via Pascoli 70/3, 20133 Milano, Italy

${ }^{b}$ Dipartimento di Chimica, Materiali e Ing. Chimica "G. Natta",

Politecnico di Milano, Piazza Leonardo da Vinci 32, 20133 Milano, Italy.

E-mail: mirella.delzoppo@polimi.it

$\dagger$ Electronic supplementary information (ESI) available. See DOI: 10.1039/ c7cp01553d
}

dispersed in a polymer matrix at low concentration, as required for example for devices with good optical transparency, the overall fluorescence emission significantly decreases.

One possible approach to increase the down conversion efficiency is that of using Metal Enhanced Fluorescence (MEF) and/or Metal Enhanced FRET (ME-FRET) obtained by incorporating metal nanoparticles (NPs). The phenomenon arises from the resonant coupling between the collective metal free electron oscillation and incident light, which produces a strong localized electromagnetic field in the proximity of metal nanoparticles (Localized Surface Plasmon (LSP)). ${ }^{17-22}$ A few experimental demonstrations of LSP enhanced FRET have been reported over the past few years. ${ }^{23-31}$ For a thorough analysis of metal enhanced FRET, it is necessary to differentiate between: (i) the direct influence of the metal NPs on the donor-acceptor luminescence and (ii) the actual effect on FRET. This is not a simple task since the exact nature of the interaction with LSPs depends on several factors, such as chemical nature, size, and shape of the metal nanoparticles ${ }^{32}$ which might, in some instances, even lead to fluorescence quenching. ${ }^{28}$ Noteworthily, the efficiency of MEF depends on the nanoparticle-fluorophore distance ${ }^{33,34}$ and most ME-FRET systems studied so far were donor/acceptor films deposited in proximity to metal NPs. ${ }^{35-37}$

In this work, we report on the preparation and optical characterization of homogeneous and transparent thin films where dodecanethiol-capped silver or gold nanoparticles are codeposited with TA in a polymethylmethacrylate (PMMA) matrix to be used as a ME-FRET system. Given the distance dependence of the plasmonic effect, the extent of TA-NP interaction is controlled by changing the relative concentrations of the two 
components and the effect is monitored by measuring the fluorescence emission. We have used both silver and gold NPs which are characterized by two different plasmon frequencies.

The best enhancement (11-fold) of the acceptor emission has been obtained with silver NPs whose plasmon is in resonance with the emission of the donor (anthracene crystal). A smaller enhancement has been obtained with gold NPs where the plasmon frequency is in resonance with the emission of the acceptor (tetracene molecules). Finally, we present the wavelength dependence of TA fluorescence emission which further elucidates the predominance of the ME-FRET.

\section{Experimental}

Unless otherwise specified, chemicals and solvents were all purchased from Sigma Aldrich and used without further purification.

\section{Synthesis of gold nanoparticles}

Gold nanoparticles (AuNPs) were prepared using the Brust two-phase method. ${ }^{38}$ In a typical procedure, $\mathrm{HAuCl}_{4}(0.6 \mathrm{mmol})$ aqueous solution $(60 \mathrm{~mL})$ was added to a tetraoctylammonium bromide TOAB $(1.2 \mathrm{mmol})$ solution in toluene $(160 \mathrm{~mL})$, and the mixture was vigorously stirred. The yellow aqueous solution became colorless, while toluene turned orange as a result of the transfer of $\left[\mathrm{AuCl}_{4}\right]^{-}$ions in the organic phase. Under vigorous stirring this solution was mixed with a solution of dodecanethiol (DT, $0.6 \mathrm{mmol}$ ) in toluene $(20 \mathrm{~mL})$ for $15 \mathrm{~min}$ at room temperature. $60 \mathrm{~mL}$ of a $0.1 \mathrm{M} \mathrm{NaBH}_{4}$ aqueous solution was added and the solution was kept under vigorous stirring for $4 \mathrm{~h}$. The organic phase was then separated and evaporated under reduced pressure. The resulting solid was heated to $180{ }^{\circ} \mathrm{C}$ with a heating rate of $2{ }^{\circ} \mathrm{C} \min ^{-1}$ and was held for $30 \mathrm{~min}$ in air. After thermal treatment the residual solid was dissolved in $35 \mathrm{~mL}$ of toluene and mixed with $400 \mathrm{~mL}$ of methanol in order to remove excess of alkanethiol and TOAB. The precipitate was filtered off, washed with methanol and redispersed in $\mathrm{CHCl}_{3}$. The average size of the nanoparticle in water was found to be $7 \mathrm{~nm}$ (see SI 01, ESI $\dagger$ for SEM images).

\section{Synthesis of silver nanoparticles}

$20 \mathrm{~mL}$ of $1 \%(\mathrm{w} / \mathrm{v})$ citrate solution and $75 \mathrm{~mL}$ of water were added in a round bottom flask and the mixture was heated to $70{ }^{\circ} \mathrm{C}$ for $15 \mathrm{~min} .1 .7 \mathrm{~mL}$ of $1 \%(\mathrm{w} / \mathrm{v}) \mathrm{AgNO}_{3}$ solution was then poured into the mixture, followed by the quick addition of $2 \mathrm{~mL}$ of $0.1 \%(\mathrm{w} / \mathrm{v})$ freshly prepared $\mathrm{NaBH}_{4}$ solution. The reaction solution was kept at $70{ }^{\circ} \mathrm{C}$ under vigorous stirring for $1 \mathrm{~h}$ and cooled to room temperature. Water was added to bring the volume of the dispersion to $100 \mathrm{~mL} .{ }^{39}$ Silver nanoparticles (AgNPs) were transferred from the aqueous phase to the organic phase by capping with dodecanethiol (DT). ${ }^{40}$ An aqueous solution of AgNPs was added to the DT-ethanol solution. The mixture was sonicated for 10-20 s to mix NPs and DT well and then left for stirring for $10 \mathrm{~min}$. The mixture was centrifuged $(14000 \mathrm{rpm}$, $10 \mathrm{~min}$ ) and the supernatant liquid (water + ethanol) was removed carefully. The precipitate was washed 2-3 times with ethanol to remove DT excess. Finally, DT-AgNPs were redispersed into chloroform. The average size of the nanoparticle in water was found to be $10 \mathrm{~nm}$. (see SI 01, ESI $\dagger$ for SEM images). Both AuNP and AgNP transfer in the organic phase leads to partial aggregation as can be seen in Fig. SI 02 (ESI $\dagger$ ) for silver NPs of different sizes.

\section{Synthesis of anthracene/tetracene doped crystals (TA)}

A chloroform solution of tetracene $(\mathrm{T})(0.5 \mathrm{mg}$ in $50 \mathrm{~mL})$ was refluxed until tetracene is dissolved to give a yellowish solution. $3 \mathrm{~g}$ of antracene (A) was added to the solution and the mixture was refluxed until A was completely dissolved. Then the reaction mixture was cooled down to $25{ }^{\circ} \mathrm{C}$. The yellow crystals obtained with a ratio of $\mathrm{A}: \mathrm{T}=7700: 1$ were filtered off.

\section{Preparation of NPs-TA thin films}

$500 \mathrm{mg}$ of PMMA and $50 \mathrm{mg}$ of TA were dissolved in $5 \mathrm{~mL}$ of $\mathrm{CHCl}_{3}$, heated at $50{ }^{\circ} \mathrm{C}$ for $2 \mathrm{~h}$ and stirred overnight at r.t. Then, different wt\% of NPs were added to the solution, and spincoated onto a pre-cleaned glass substrate at $2000 \mathrm{rpm}$ for $2 \mathrm{~min}$ by a Laurell WS-400-6NPP-LITE; (samples are listed in Table SI 01, $\mathrm{ESI} \dagger$ ). For a comparison, films with the A donor and the $\mathrm{T}$ acceptor were prepared with the same concentrations without NPs as the TA films.

\section{Film characterization}

The optical images of the samples were recorded using an Olympus BX41 microscope and a 50× objective. The UV-vis absorption spectra were recorded using a Varian Cary 5000 spectrometer. The photoluminescence spectra were recorded using a JASCO FP 6600 spectrofluorometer. Field emission SEM Zeiss SUPRA 40 was used for scanning electron microscopy. The ImageJ software was used to measure the average size of the NPs and the TA from SEM images.

\section{Results and discussion}

In our previous work ${ }^{16}$ we reported the preparation and the characterization of TA-PMMA films for LSCs. The devices prepared with a high concentration of TA in the matrix $(\mathrm{A}: \mathrm{T}=250: 1)$ show good luminescence properties but poor optical transparency. The most performing TA-PMMA film consists of large TA crystals (about one-hundred-micron size) randomly oriented in the polymer matrix.

In this work, with the purpose of improving the transparency of the films, we used a much lower TA/polymer concentration and a much lower ratio of the active species along with NPs. Films with a better transparency and overall improved optical quality have been obtained (see Fig. 1). Indeed, very small pale yellow TA-crystals (size of hundreds nanometers) were formed which are more suitable to be used as an active layer in devices where good optical properties and transparency are mandatory. Polystyrene was also tested as a polymer matrix giving similar results.

Different morphologies are obtained with or without NPs (Fig. 1), clearly indicating that significant changes are induced 

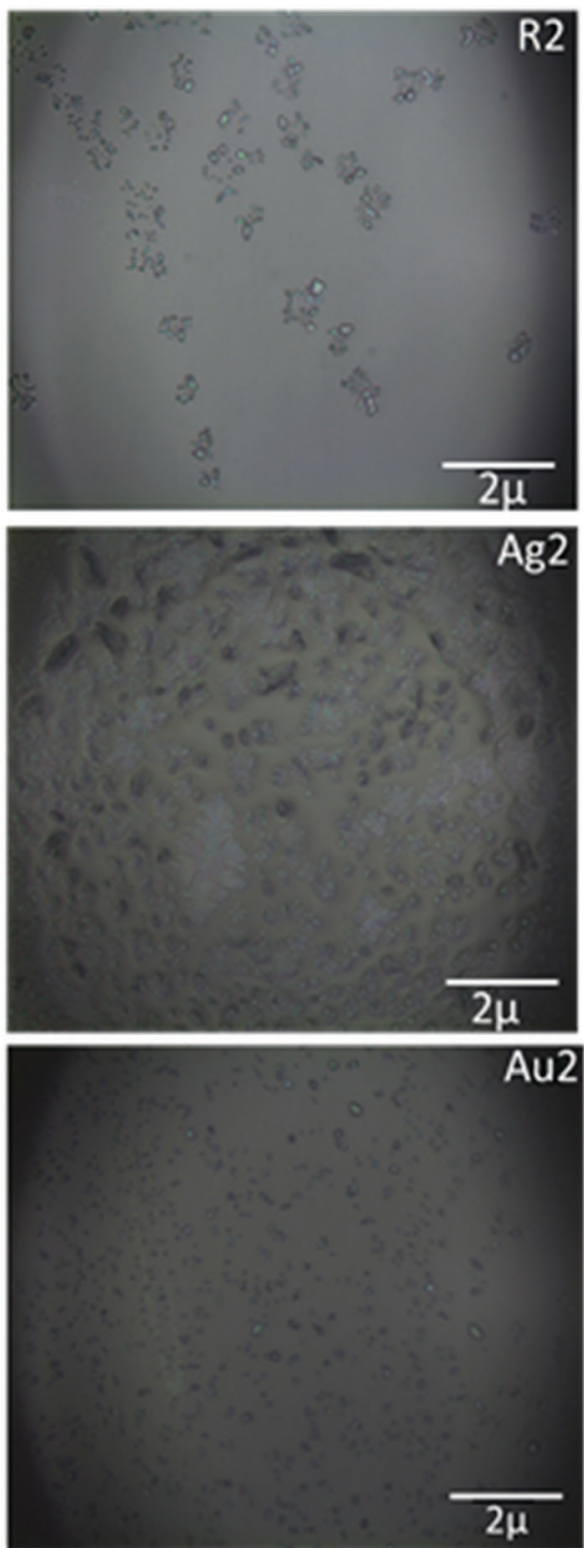

Fig. 1 Optical microscope images (objective magnification $50 \times$ of TA/PMMA films: without NPs (R2), with AuNPs (Au2) and with AgNPs (Ag2)). See details on sample composition in the ESI.†

by the addition of NPs even at low concentration (see Table SI 01, $\mathrm{ESI} \dagger$ ). In the reference samples (R2) i.e. PMMA films without NPs and with the same relative polymer/TA concentration, many isolated crystals grow with a dimension of hundreds of nanometers.

In contrast, in the presence of both gold and silver nanoparticles, a higher number of smaller crystals are observed, thus, suggesting that the nanoparticles can act as nucleating agents. Smaller crystal dimensions, with respect to those of ref. 16, also mean an overall more homogeneous distribution of TA in the matrix, which improves the optical quality of the films. In Fig. 2a, we report the UV-vis absorption spectrum of the TA film (blue line) which reveals the predominant contribution of isolated A species. Indeed, the well-resolved vibronic structure

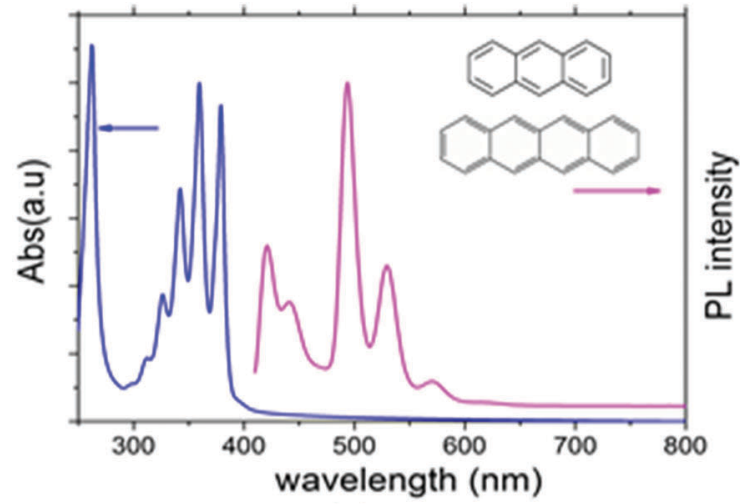

(a)

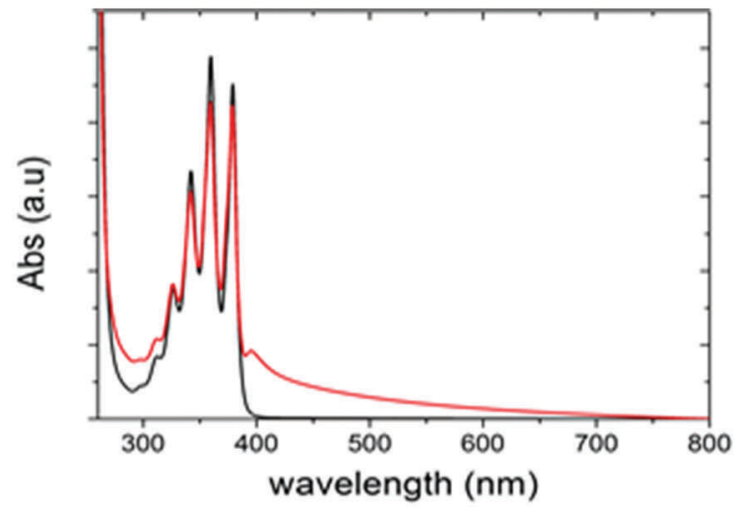

(b)

Fig. 2 (a) UV-vis absorption (blue curve) and PL (pink curve) spectra of the TA/PMMA film. Excitation line: $400 \mathrm{~nm}$. In the inset: anthracene and tetracene structures. (b) Absorption spectra of TA in PMMA films without NPs (black curve), with AgNPs (red curve).

with peaks at $341 \mathrm{~nm}, 359 \mathrm{~nm}$, and $379 \mathrm{~nm}$ is assigned to the absorption of isolated A species dispersed in PMMA as can be seen from the comparison with the absorption spectrum of anthracene in $\mathrm{CHCl}_{3}$ solution (see SI 03, ESI $\dagger$ ).

The absorption spectrum of TA in the PMMA matrix at a concentration much higher than that used in Fig. $2 \mathrm{a}^{16}$ shows a peak at $400 \mathrm{~nm}$ which can be ascribed to crystalline A (see SI 03, ESI $\dagger$ ). This peak, which shows a negligible intensity in the R2 films, becomes appreciable when metal NPs are added (Fig. 2b). No distinct visible absorption peaks of isolated (see SI 04, ESI $\dagger$ ) or aggregated $\mathrm{T}$ can be observed because of its extremely low concentration. The absorption spectra of colloidal silver and gold nanoparticles in the aqueous/organic phase are reported in SI 05 and SI 06 (ESI $\dagger$ ) along with their respective spectra in the PMMA matrix. In the polymer matrix, the intensity of plasmon absorption is very low, with a red-shift and broadening of the peak due to partial NP aggregation (see SI 05 and SI 06, ESI $\dagger$ ).

The PL spectrum of the TA film (reported in Fig. 2a) shows the emission of both $\mathrm{A}$ and $\mathrm{T}$. Upon excitation at $400 \mathrm{~nm}$, only crystalline A can absorb the incident photons and we mainly observe emission at $443 \mathrm{~nm}$ (see SI 07, ESI $\dagger$ ). Only when the samples are excited at a higher energy $(360 \mathrm{~nm})$, we can have emission from isolated A species as can be seen in Fig. SI 07 (ESI $\dagger$ ) 
where the shoulder at $455 \mathrm{~nm}$ is assigned to a component of the progression of vibronic transitions typical of isolated molecules.

Noteworthily, $\mathrm{T}$ emission is intense in spite of its extremely low concentration, confirming the existence of an efficient FRET from donor to acceptor species in TA embedded in the PMMA matrix. It is known that in pure TA crystals FRET efficiency is very high and at a relatively high $\mathrm{T}$ concentration (i.e. $\mathrm{A}: \mathrm{T}=250: 1)$ no $\mathrm{A}$ emission can be observed in the PL spectra. ${ }^{5,16}$ However, in present experiments working at lower $\mathrm{T}$ concentrations, A emission is still visible.

Since it is known that metal NPs can increase the Förster radius $^{28}$ and hence the amount of energy transfer, we added gold and silver NPs to the different systems, namely A only, T only (in the same concentration used in TA) and TA, and we further analyzed the emissions.

In the case of the donor (A)/PMMA film upon the addition of NPs (both gold and silver) we observe in the absorption spectrum a clear peak at $400 \mathrm{~nm}$ which corresponds to crystalline A (see SI 08, ESI $\dagger$ ) and an increased PL. In the case of acceptor (T)/ PMMA films we do not observe either absorption or emission peaks even upon the addition of NPs because of the extremely low $\mathrm{T}$ content (see SI 09, ESI $\dagger$ ).

In the TA case, the addition of NPs to the PMMA film increases the overall emission significantly. We observe a large increase in the $\mathrm{T}$ emission and a smaller increase in the $\mathrm{A}$ emission with respect to the reference samples, as reported in Fig. 3; this evidence seems to suggest that the more efficient down-conversion is due to a nanoparticle-mediated energy transfer phenomenon.

It is well known that there is an optimal fluorophore metal-NP distance for maximum enhancement. ${ }^{19,34,41-44}$ In order to examine the dependency of TA-NP interaction on their mutual average distance, we have prepared blends with different NP concentrations. To obtain the enhancement factor of $\mathrm{T}$ in TA films with NPs, fluorescence spectra have been recorded using the excitation wavelength at $400 \mathrm{~nm}$. All the values have been obtained using the integrated area (470-650 $\mathrm{nm}$ ) of the PL spectra and were averaged over a set of measurements taken in different sample regions. To have an effective comparison of the enhancement,

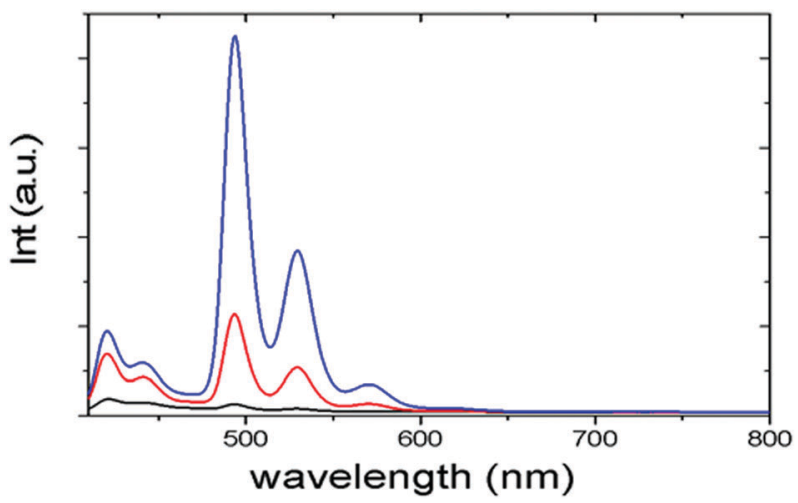

Fig. 3 Emission spectra of TA in PMMA films without NPs (black curve), with AuNPs (red curve) and with AgNPs (blue curve) at maximum enhancement (1.66 NP wt\% for silver and 0.9 NP wt\% for gold) (Exc: $400 \mathrm{~nm}$ ).
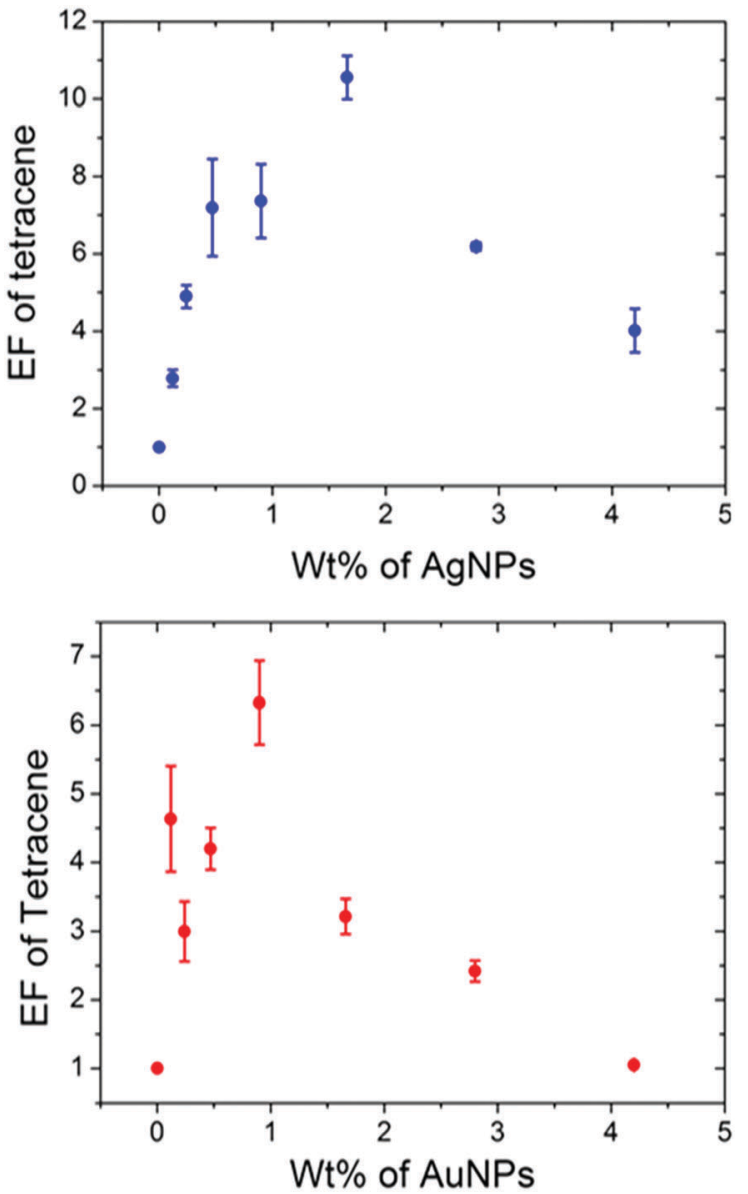

Fig. 4 Enhancement factors of T emission for TA-NPs films with varying AgNP and AuNP concentrations.

all the integrated values of the samples were normalized over the respective reference sample (without NPs).

Fig. 4 shows that, for silver nanoparticles whose plasmon absorption is in resonance with the donor (A) emission (SI 10, $\mathrm{ESI} \dagger$ ), a maximum 11-fold enhancement is achieved at $1.66 \mathrm{wt} \%$ of AgNPs (spectrum reported in Fig. 3). For TA films with gold nanoparticles, which have a plasmon in resonance with the acceptor emission (SI 10, ESI $\dagger$ ), a maximum 6.5-fold enhancement is obtained at $0.90 \mathrm{wt} \%$ of AuNPs (spectrum also reported in Fig. 3).

In order to rationalize the observed enhancement, we can note that the presence of nanoparticles not only alters the excitation rate of both the donor (A) and the acceptor (T) but also changes the rates of the various decay channels (i.e., energy transfer from the donor to the acceptor, energy transfer between the plasmon resonance and the fluorophores, the radiative decay to the far field and the nonradiative decay). As discussed in the literature, ${ }^{45,46}$ it is very difficult to discriminate among the various effects; nevertheless, a few general considerations can be done.

In the presence of gold NPs, the excitation line (400 nm) is not in resonance with the plasmon absorption $(550 \mathrm{~nm})$. Notwithstanding, we do observe an enhancement of both donor 
(with or without acceptor) and acceptor emissions. $\mathrm{ID}_{\mathrm{A}}$ and ID (which are, respectively, the donor emission intensity in the presence or absence of acceptor) increase by the same amount and hence, the FRET efficiency, which is defined as $E_{\mathrm{FRET}}=1-\mathrm{ID}_{\mathrm{A}} / \mathrm{ID}$, does not change. The increase in the emission intensity can therefore be associated with the formation of a larger number of crystalline domains (see Fig. 1), which are necessary for FRET to occur. Moreover, $\mathrm{T}$ emission is further enhanced because of the resonance with the plasmon.

In the case of silver nanoparticles, the excitation wavelength is in resonance with plasmon absorption which overlaps with the donor (A) emission and the acceptor (T) absorption (see SI 10, ESI $\dagger$ ). Under these conditions, the FRET rate should increase (see for example ref. 30). However, decreased FRET efficiency was observed in spite of the increase in the FRET rate. Actually, the energy transfer efficiency E defines the fraction of the energy transfer event occurring per donor excitation event according to $E=k_{\mathrm{ET}} /\left(k_{\mathrm{D}}+k_{\mathrm{ET}}\right)$ where $k_{\mathrm{D}}$ is the sum of the radiative and nonradiative decay rates of the donor. ${ }^{30}$ When the increase in the donor rate is larger than that of the FRET, the efficiency decreases. This seems to be the case with silver NPs (for which we obtain from the data in Table SI 02 (ESI $\dagger$ ) a decrease in efficiency from 45 to $38 \%$ ). Indeed, the intensity of the emission of the donor in the presence of the acceptor increases more than the intensity in the donor only sample $\left(\mathrm{ID}_{\mathrm{A}}{ }^{\prime} / \mathrm{ID}_{\mathrm{A}}=4.1 \mathrm{vs}\right.$. $\mathrm{ID}^{\prime} / \mathrm{ID}=3.6$ as seen in Table SI 02, ESI $\dagger$ ). Additionally, as already seen in the case of gold, there is an effect on emission due to the larger number of crystalline domains.

The larger enhancement obtained with silver NPs suggests that plasmon resonance affects FRET to a larger extent with respect to metal-enhanced emission of $\mathrm{T}$. Indeed, even in resonance with $\mathrm{T}$ emission (as is the case for AuNP) the maximum enhancement is lower than that obtained with AgNPs.

To further elucidate the mechanisms of plasmon/TA interaction, the excitation wavelength dependent study of $\mathrm{T}$ emission has been carried out on the TA-NPs films that exhibit the maximum enhancement. Fig. 5 shows the T fluorescence intensity for samples with AuNPs (red) and AgNPs (black) excited at different wavelengths; all the values obtained are normalized with respect to the corresponding reference sample. With the

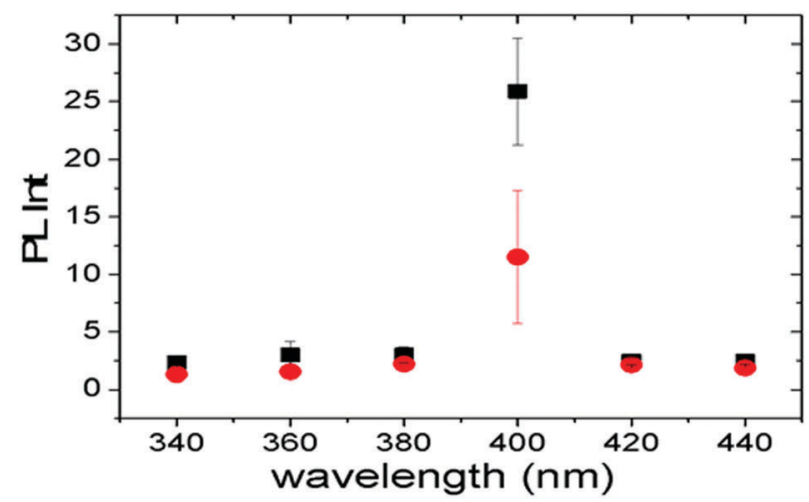

Fig. $5 \mathrm{PL}$ enhancement of T emission in TA films with AgNPs (black) and with AuNPs (red) excited at different wavelengths. addition of nanoparticles (both silver and gold NPs) $\mathrm{T}$ emission increases and reaches a maximum at $400 \mathrm{~nm}$ when the excitation line is in resonance with the absorption of crystalline A. Below $400 \mathrm{~nm}$ we are mainly exciting isolated A species which do not contribute to FRET (hence small $\mathrm{T}$ emission). This clearly confirms the role of the crystalline fraction in promoting the NP mediated energy transfer FRET in TA.

\section{Conclusions}

We have shown that it is possible to obtain transparent films with good optical properties and an efficient energy transfer by using a host guest anthracene/tetracene crystalline system embedded in a polymer matrix in the presence of metal nanoparticles with an appropriate relative NP/TA concentration. This system can be used in optical devices where efficient down-conversion (about $100 \mathrm{~nm}$ ) of incident radiation is required. The enhancement induced by the presence of silver NPs can be as large as 11 and it is due to several concurring phenomena: first of all, metal NPs act as nucleating agents and hence increase the number of small TA crystals which take part in the FRET process. Moreover, in the case of silver, excitation of the plasmon resonance increases the FRET rate and leads to a further increase of the acceptor emission.

\section{Conflicts of interest}

There are no conflicts of interest to declare.

\section{Acknowledgements}

We gratefully acknowledge meaningful contribution by Giorgio Pariani in data discussion and processing.

\section{References}

1 F. Gao, Q. Liao, Z.-Z. Xu, Y.-H. Yue, Q. Wang, H.-L. Zhang and H.-B. Fu, Angew. Chem., Int. Ed., 2010, 49, 732-735.

2 C. A. Strassert, C.-H. Chien, M. D. G. Lopez, D. Kourkoulos, D. Hertel, K. Meerholz and L. De Cola, Angew. Chem., Int. Ed., 2011, 50, 946-950.

3 Q. Feng, M. Wang, B. Dong, C. Xu, J. Zhao and H. Zhang, CrystEngComm, 2013, 15, 3623-3629.

4 H. Wang, F. Li, B. Gao, Z. Xie, S. Liu, C. Wang, D. Hu, F. Shen, Y. Xu, H. Shang, Q. Chen, Y. Ma and H. Sun, Cryst. Growth Des., 2009, 9, 4945-4950.

5 H. Wang, B. Yue, Z. Xie, B. Gao, Y. Xu, L. Liu, H. Sun and Y. Ma, Phys. Chem. Chem. Phys., 2013, 15, 3527-3534.

6 S. K. Park, S. Varghese, J. H. Kim, S.-J. Yoon, O. K. Kwon, B.-K. An, J. Gierschner and S. Y. Park, J. Am. Chem. Soc., 2013, 135, 4757-4764.

7 L. Viani, L. P. Tolbod, M. Jazdzyk, G. Patrinoiu, F. Cordella, A. Mura, G. Bongiovanni, C. Botta, D. Beljonne, J. Cornil, M. Hanack, H.-J. Egelhaaf and J. Gierschner, J. Phys. Chem. B, 2009, 113, 10566-10570. 
8 Y. S. Zhao, H. Fu, F. Hu, A. Peng, W. Yang and J. Yao, Adv. Mater., 2008, 20, 79-83.

9 X. Wu, M. Wang, M. Du, J. Lu, J. Chen, A. Khan, R. Usman, X. Wei, Q. Feng and C. Xu, Cryst. Growth Des., 2015, 15, 434-441.

10 A. Del Guerzo, A. G. L. Olive, J. Reichwagen, H. Hopf and J. P. Desvergne, J. Am. Chem. Soc., 2005, 127, 17984-17985.

11 Q. Feng, M. Wang, C. Xu, A. Khan, X. Wu, J. Lu and X. Wei, CrystEngComm, 2014, 16, 5820-5826.

12 T. Gatti, L. Brambilla, M. Tommasini, F. Villafiorita-Monteleone, C. Botta, V. Sarritzu, A. Mura, G. Bongiovann and M. Del Zoppo, J. Phys. Chem. C, 2015, 119, 17495-17501.

13 L. Beverina and A. Sanguineti, Organic Fluorophores for Luminescent Solar Concentrators, John Wiley \& Sons, Inc., 2013, pp. 317-355.

14 C. Botta, P. Betti and M. Pasini, J. Mater. Chem. A, 2013, 1, 510-514.

15 M. J. Currie, J. K. Mapel, T. D. Heidel, S. Goffri and M. A. Baldo, Science, 2008, 321, 226-228.

16 G. Griffini, L. Brambilla, M. Levi, C. Castiglioni, M. Del Zoppo and S. Turri, RSC Adv., 2014, 4, 9893-9897.

17 C. D. Geddes and J. R. Lakowicz, Editorial Metal-Enhanced Fluorescence, J. Fluoresc., 2002, 12, 121-129.

18 J. R. Lakowicz, K. Ray, M. Chowdhury, H. Szmacinski, Y. Fu, J. Zhang and K. Nowaczyk, Analyst, 2008, 133, 1308-1346.

19 H. Mishra, B. L. Mali, J. Karolin, A. I. Dragan and C. D. Geddes, Phys. Chem. Chem. Phys., 2013, 15, 19538-19544.

20 J. Zhang and J. R. Lakowicz, Opt. Express, 2007, 15, 2598-2606.

21 W. Zhong, Anal. Bioanal. Chem., 2009, 394, 47-59.

22 P. P. Pompa, L. Martiradonna, A. Della Torre, F. Della Sala, L. Manna, M. De Vittorio, F. Calabi, R. Cingolani and R. Rinaldi, Nat. Nanotechnol., 2006, 1, 126-130.

23 J. Zhang, Y. Fu and J. R. Lakowicz, J. Phys. Chem. C, 2007, 111, 50-56.

24 M. L-Viger, D. Brouard and D. Boudreau, J. Phys. Chem. C, 2011, 115, 2974-2981.

25 M. Lunz, X. Zhang, V. A. Gerard, Y. K. Gun'ko, V. Lesnyak, N. Gaponik, A. S. Susha, A. L. Rogach and A. L. Bradley, J. Phys. Chem. C, 2012, 116, 26529-26534.

26 X. Zhang, C. A. Marocico, M. Lunz, V. A. Gerard, Y. K. Gun'ko, V. Lesnyak, N. Gaponik, A. S. Susha, A. L. Rogach and A. L. Bradley, ACS Nano, 2014, 8, 1273-1283.
27 P. C. Ray, Z. Fan, R. A. Crouch, S. S. Sinha and A. Pramanik, Chem. Soc. Rev., 2014, 43, 6370-6404.

28 M. Lunz, V. A. Gerard, Y. K. Gun'ko, V. Lesnyak, N. Gaponik, A. S. Susha, A. L. Rogach and A. L. Bradley, Nano Lett., 2011, 11, 3341-3345.

29 R. G. West and S. M. Sadeghi, J. Phys. Chem. C, 2012, 116, 20496-20503.

30 L. Zhao, T. Ming, L. Shao, H. Chen and J. Wang, J. Phys. Chem. C, 2012, 116, 8287-8296.

31 X. Zhao, P. Wang and B. Li, Nanoscale, 2011, 3, 3056-3059. 32 J. R. Lakowicz, Anal. Biochem., 2005, 337, 171-194.

33 L. Bene, G. Szentesi, L. Matyus, R. Gaspar and S. Damjanovich, J. Mol. Recognit., 2005, 18, 236-253.

34 E. V. Canesi, M. Capsoni, L. Karnam, A. Lucotti, C. Bertarelli and M. Del Zoppo, J. Phys. Chem. C, 2013, 117, 13197-13201.

35 V. K. Komarala, A. L. Bradley, Y. P. Rakovich, S. J. Byrne, Y. K. Gun'ko and A. L. Rogach, Appl. Phys. Lett., 2008, 93, 123102.

36 C. H. Wang, C. W. Chen, Y. T. Chen, C. M. Wei, Y. F. Chen, C. W. Lai, M. L. Ho, P. T. Chou and M. Hofmann, Appl. Phys. Lett., 2010, 96, 071906.

37 X.-R. Su, W. Zhang, L. Zhou, X.-N. Peng, D.-W. Pang, S.-D. Liu, Z.-K. Zhou and Q.-Q. Wang, Appl. Phys. Lett., 2010, 96, 043106.

38 M. Brust, M. Walker, D. Bethell, D. J. Schiffrin and R. Whyman, J. Chem. Soc., Chem. Commun., 1994, 801-802.

39 Y. Wan, Z. Guo, X. Jiang, K. Fang, X. Lu, Y. Zhang and N. Gu, J. Colloid Interface Sci., 2013, 394, 263-268.

40 X. Wang, S. Xu, J. Zhou and W. Xu, J. Colloid Interface Sci., 2010, 348, 24-28.

41 Metal-enhanced fluorescence, ed. C. D. Geddes, Wiley-Blackwell, Oxford, 2010.

42 J. Zhang, Y. Fu, M. H. Chowdhury and J. R. Lakowicz, J. Phys. Chem. C, 2007, 111, 11784-11792.

43 A. I. Dragan, E. S. Bishop, J. R. Casas-Finet, R. J. Strouse, J. McGivney, M. A. Schenerman and C. D. Geddes, Plasmonics, 2012, 7, 739-744.

44 P. Anger, P. Bharadwaj and L. Novotny, Phys. Rev. Lett., 2006, 96, 113002.

45 R. G. West and S. M. Sadeghi, J. Phys. Chem. C, 2012, 116, 20496-20503.

46 P. Ghenuche, M. Mivelle, J. de Torres, S. B. Moparthi, H. Rigneault, N. F. Van Hulst, M. F. Garcia-Parajo and J. Wenger, Nano Lett., 2015, 15, 6193-6620. 